\title{
A combined analysis of plant connectivity and alarm logs to reduce the number of alerts in an automation system
}

\author{
Markus Schleburga,b ${ }^{a}$, Lars Christiansen ${ }^{a}$, Nina F. Thornhill ${ }^{\mathrm{b}, *}$, Alexander Fay ${ }^{\mathrm{a}}$ \\ a Institute of Automation Technology, Helmut-Schmidt-University/University of the Federal Armed Forces, Holstenhofweg 85, D-22043 Hamburg, Germany \\ ${ }^{\mathrm{b}}$ Centre for Process System Engineering, Department of Chemical Engineering, Imperial College London, London SW7 2AZ, UK
}

\section{A R T I C L E I N F O}

\section{Article history:}

Received 16 September 2012

Received in revised form 27 January 2013

Accepted 27 March 2013

\section{Keywords:}

Alarm flood

Alarm management

ISA 18.2

EEMUA 191

Rule-based systems

IEC 62424.

\begin{abstract}
A B S T R A C T
A process alarm arises when normal operation limits are exceeded and an alarm management system alerts the operator of a process plant. Due to the material, energy and information flow in a plant, single disturbances can cause multiple consequent alarm messages, and the alarm messages may overload the operator by presenting many redundant alarms. This undesired situation is called an 'alarm flood'. In such situations, the operator might not be able to fulfil his required tasks to keep the plant within safe operation limits and to find the root cause of the disturbance. The aim of the work presented in this paper is to reduce the number of alerts presented to the operator. If alarms are related to one another, those alarms should be grouped and presented as one alarm problem. For the implementation of the concept, a software prototype has been developed to perform this reduction automatically. The analysis process starts with the alarm history which is a log containing all past alarm messages. This is combined with the plant topology of the controlled system and a set of rules. The rules describe typical interrelations between alarm messages which have a common cause. The combination of these three elements yields an effective alarm management strategy that can help plant owners and operators to comply with standards for alarm management such as ANSI/ISA 18.2 (2009) and EEMUA 191 (2007) which set limits on the number of alarms per unit time for an operator. The effectiveness of the approach is illustrated by two industrial examples where a significant reduction of alarms has been achieved.
\end{abstract}

(c) 2013 Elsevier Ltd. All rights reserved.

\section{Introduction}

\subsection{Motivation}

Large chemical, refining, power generation and other processing plants require the use of an automation system to keep the process operating successfully within the boundaries specified by the process designers. Exceeding these boundaries might endanger the quality of the product, the stability of the process, or even the safety of the plant. Therefore, the operators in the control room are notified by alarm messages when certain alarm limits are violated, in order to react properly. In the case of a plant disturbance, usually several process values (such as pressure, level, flow, temperature) are affected simultaneously. The operator is confronted with several alarm messages within a short time interval, and might not be able to react appropriately. Therefore, alarm management concepts are under development which aim at a reduction of the number of separate alarms.

\footnotetext{
* Corresponding author. Tel.: +44 2075946622.

E-mail address: n.thornhill@imperial.ac.uk (N.F. Thornhill).
}

Industrial plants are highly automated systems and are continuously monitored to avoid plant shut-downs which lead to a reduced plant availability combined with substantial costs, and to avoid environmental damage and/or human injuries. Therefore different monitoring techniques are applied to indicate abnormal behaviour of the plant and to detect faults [1]. Abnormal behaviour usually results in the deviation of at least one process variable from setpoint. A severe deviation results in a violation of an alarm limit value and, thus, produces an alarm. This alarm information will be notified to the operator as an alarm message. The operator has to react immediately to bring the plant into a safe state and to find the root cause of this alarm.

A key step in managing an alarm scenario is causal analysis of the alarm list, which may show several or many alarms for each incident, using resources such as piping and instrumentation diagrams or equipment manuals. The task of analyzing the alarm list has been the focus of much attention from researchers and automation companies driven by initiatives such as the Abnormal Situation Management ${ }^{\circledR}$ consortium (http://www.asmconsortium.net/).

An industrial automation system represents the process in a way that does not easily lend itself to causal analysis. As an example, the upper left hand of Fig. 1 shows an operator graphic for a batch reactor created by one of the authors during a training course. The list 


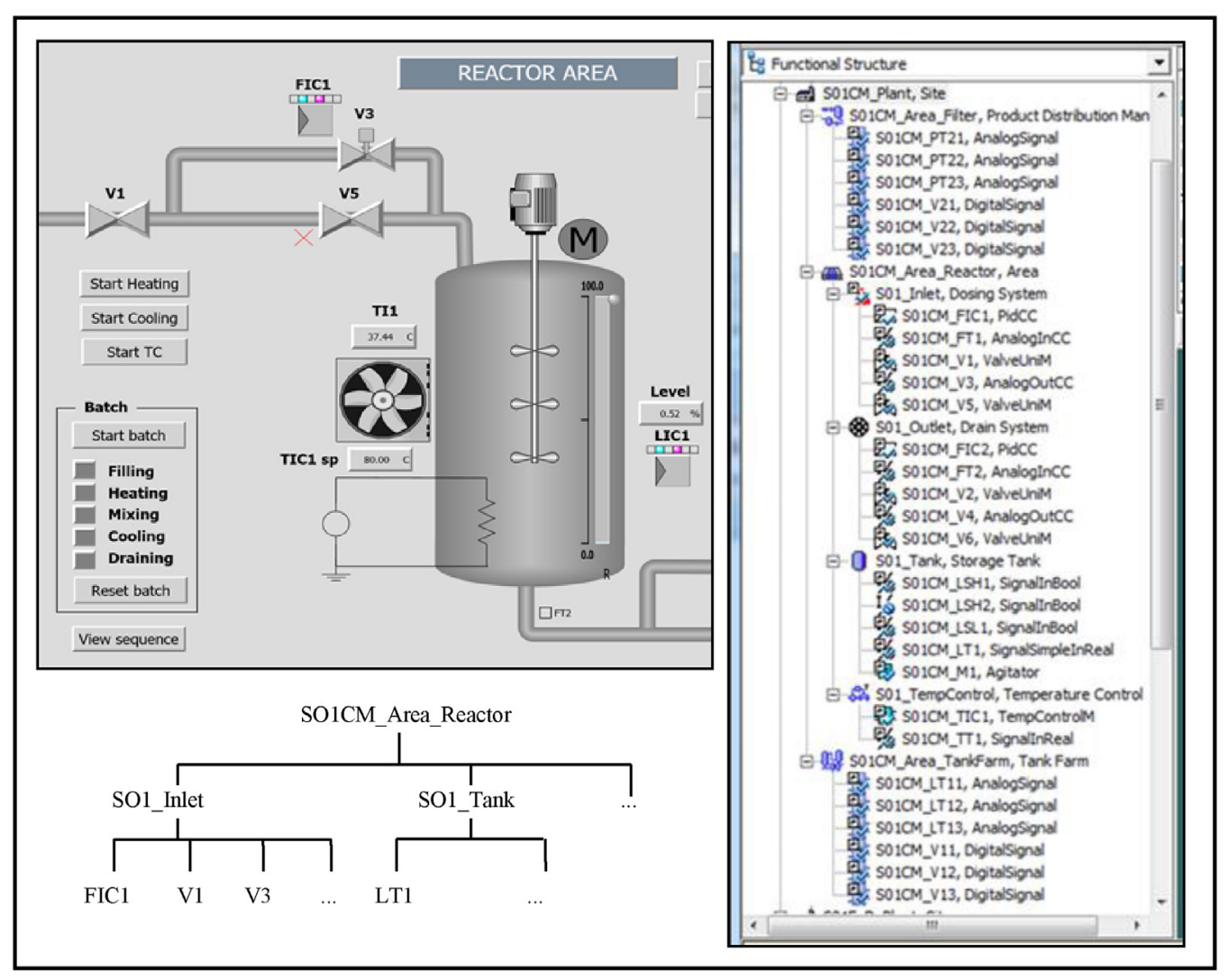

Fig. 1. Representation of a process within a process automation system.

on the right shows how the functional structure of the process is represented in the automation system. It describes the hierarchy illustrated in the lower left of Fig. 1 which shows FIC1 is part of the Inlet Dosing system along with FT1, V1, V3 and other items of equipment. Likewise, the level sensor LT1 is part of the Storage Tank system. It can be inferred from the operator graphic that opening V1 and V3 will cause a change in LT1. Indeed, leaving V1 and V3 open for too long obviously has the potential to cause a high level alarm. However, information about the physical causal relationship between the flow indicated by FIC1 and the level indicated by LT1 is not present in the hierarchical functional structure of the automation system. What is needed is an electronic representation of the causal connection between FIC1 and LT1 through the process. This paper demonstrates how the necessary causal information about the process can be captured in electronic form, combined with alarm information from the automation system and analyzed using insightful interrelation rules.

The next section of the paper outlines the motivations for the work. It reviews methods for alarm management and alarm handling, and discusses ways to make use of the causal relationships in the process. Section 3 explains tools for capture of the connectivity structure of a process plant from its piping and instrumentation diagram. Section 3 also introduces a case study, and Section 4 discusses the alarm logs from the automation system of the case study. The novel approach of this paper is to apply causal relationships and simple physical insights to the problem of clustering alarms into related groups in order to simplify the task of diagnosis. Sections 6 and 7 discuss implementation and testing of a software prototype that provides a menu-driven graphical user interface to discover which alarms are causally related. The results show that grouping alarms according to causal relationships in the process gives a significant reduction in the number of alarms an operator has to handle. The paper closes with discussion and conclusions.

\subsection{Related works}

Various fault detection methods have been implemented as a result of the continuous rising influence of digital technology and it is possible to monitor each single process variable [2]. This results in a high quantity of information presented to the operator. Alarm systems which are badly designed, badly tuned or which present too much information to the operator can lead to nuisance alarms. As a result, the operator may have to acknowledge alarms without responding to them which leads to the problem that the operator may not recognize potentially hazardous states. As discussed, a process has connections for product and energy transport, deviations and disturbances are propagated through it. This circumstance causes more deviations at other parts of the facility, and depending on the alarm thresholds, raises further alarms. This causal relationship can produce alarm floods. For instance, a single compressor failure can lead to numerous pressure, temperature or flow rate alarms, which will be consecutively displayed without a hint to the operator that they are related to each other.

Standards and guidelines have been developed and published for preventing the aforementioned alarm flood scenarios, giving hints for an effective alarm management design during the engineering process. As a general guideline for designing alarm management systems for process facilities, the standards ISA 18.2 [3] and EEMUA 191 [4] define a general method for designing alarm systems. Furthermore they state that an alarm rate of just one alarm per 10 min should not be exceeded in normal operation. However, in reality this value is rarely achieved.

Several approaches have been proposed to minimize the number of alarms. Adnan et al. [5] focused on bad actors by the fact that the most alarms are caused by just a small number of variables or actors (80-20-rule). The idea is to use different data bases which are generated by the alarm system such as alarm logs and process 
history data combined with process knowledge. Therefore, quantitative models for physical behaviour, heuristic knowledge based on operational experience, and connectivity information like process flow diagrams or piping and instrumentation diagram (P\&ID) are used. Other methods to reduce the number of alarms include suppression of individual alarms by techniques such as delay-timers, deadband and alarm filtering. The performance of these methods has been analyzed by Adnan et al. [5]. Ahnlund and Berquist [6] presented a clean-up methodology with a tool-supported approach for reducing the number of nuisance alarms in a facility by using signal processing methods and predictive algorithms. Candy and Taisne [7] introduced an expert system that creates patterns from process data to identify alarm series. The system combined all found alarm messages into a single diagnosis message. Another approach which uses alarm logs as a basis for reduction of alarm floods is discussed in Aizpurúa [8]. In this approach, it is assumed that the first alarm in a larger series of alarms is the alarm root cause. Actually, this assumption does not always have to be true due to several influences: (1) alarm limits might have been fixed at different values, causing alarms to rise in an order different from the physical causes and (2) physical effects like varying propagation speeds of temperature and pressure depending on liquid or steam products can cause alarms to propagate and rise in different order.

Gabbar et al. [9] defined fault models and searched for fault propagation paths by a plant topology analysis. This analysis is used to find automatically all possible propagation paths. A fault library defined a model for every possible plant failure with involved process types and devices. The application was also able to visualize the propagation path on the P\&ID to help the operator in finding the cause for the plant failure.

Recently, process data diagnosis has been combined with plant topology analysis to improve the root cause analysis of plant-wide disturbances. The idea is that process data analysis methods show possible root causes for a series of alarms. The plant topology can be used afterwards to verify these assumptions, by revealing a physical or functional connection between a number of given alarms. The root cause is assumed to be found upstream of the process flow direction [10]. In that work, a connectivity matrix was created from an XML (Extensible Markup Language) model which describes the plant structure [11]. An algorithm determined if a connection between two disturbed plant devices can be found. The authors found that a combination of process data and topology information resulted in more reliable root cause identification.

As highlighted in [12], it is possible for an alarm system to hinder rather than help an operator to prevent or minimize the consequences of faults. This observation gives a motivation for properly designed and maintained alarm systems. The industrial state of the art includes benchmarking of alarm rates against standards, help with alarm configuration, alarm filtering and hiding, and sharing of alarms with a remote support centre. A typical industrial alarmhiding function removes alarms from the alarm list that need no action from the operator at the present time. They reflect the functional structure of the process such as is shown in the lower left of Fig. 1. For instance, all alarms related to a pump might be hidden when the pump is in standby mode. Other alarms would be hidden during startup and shutdown when process variables are expected to be out of their normal range. There are good practice guidelines and standards for design of alarm management systems. They describe a complete alarm system lifecycle with all necessary steps which have to be taken to achieve and maintain a responsible alarm system. Techniques to handle chattering alarms have been developed and the ability to shelve alarms assists the plant operator. Several case studies have shown success with advanced alarm management techniques, involving alarm history, process data and plant topology (e.g. $[13,14])$. However, the situation in many production facilities still requires improved methods to reduce the number of alarms presented to the operator. Within this paper, the authors present a new idea for analyzing the available data resources. The components of the proposed alarm analysis system comprise a description of plant topology in the form of a process schematic or P\&ID (Pipe and Instrumentation Diagram), a process alarm log, and a set of interrelation rules which specify when alarms can be grouped.

\section{Modelling of the plant topology}

\subsection{Computer Aided Engineering eXchange (CAEX)}

P\&I-Diagrams (P\&IDs) provide a model of the items of a plant. They describe the physical and functional items of a plant and their dependencies in a graphical manner, showing material flow and information flow.

The graphical representation must be available in a computeraccessible format for effective use of this model for alarm management purposes. Object-oriented representations of processes and plants are becoming widely available with modern CAD tools. P\&IDs can now be exported into vendor independent and XML-based data formats, giving a portable text file that describes all relevant equipments, their properties and the connections between them. The representation of the model in the vendor independent data exchange format CAEX (Computer Aided Engineering eXchange) which is standardized in IEC 62424 [11] makes it possible to transfer the content of the P\&ID into a topology model of the plant. CAEX was developed as an object-oriented data model with features such as libraries, re-use and inheritance $[15,16]$.

CAEX is based on a fourfold concept which comprises three libraries (RoleClassLib, InterfaceClassLib, SystemUnitClassLib) and an InstanceHierarchy.

The RoleClassLib provides abstract objects for defining the functionality of an object. Every item of the plant has a reference to a role to enable the semantic interpretation of items during the exchange. The InterfaceClassLib is a library to define types of the interfaces for material, energy or information transport. A distinction is made between product and signal connections. A product connection represents a pipe which allows the material flow from one item to another. A signal connection describes connections of the control system between two elements, for example between a sensor and a valve. A SystemUnitClass describes a more specific template of a plant device with its type of interfaces. The SystemUnitClassLib allows insertion of new instances (items) of each SystemUnitClass into the InstanceHierarchy, which are represented as InternalElements (IE). CAEX forms the backbone concept of the data exchange format AutomationML (AML), which has been developed for supporting the data exchange between heterogeneous engineering tools for large engineering projects.

\subsection{CAEX plant topology model}

Every plant which can be described by a P\&ID can be modelled in Computer Aided eXchange format (CAEX). The method to transform P\&ID content into a CAEX model is described in detail in [17].

This CAEX model contains the physical and logical interrelationships between all items in the facility, for example vessels, compressors, valves and sensors. The product connection between a compressor and a vessel represents a physical connection with internal product flow. In contrast to this, a signal connection between sensor and valve describes a control system connection. Thus, the topology model describes all plant devices with all interconnections between them. Fig. 2 shows one section of a plant while Fig. 3 illustrates the same plant section as a hierarchical model in CAEX format, shown in the AutomationML-Editor [18]. 


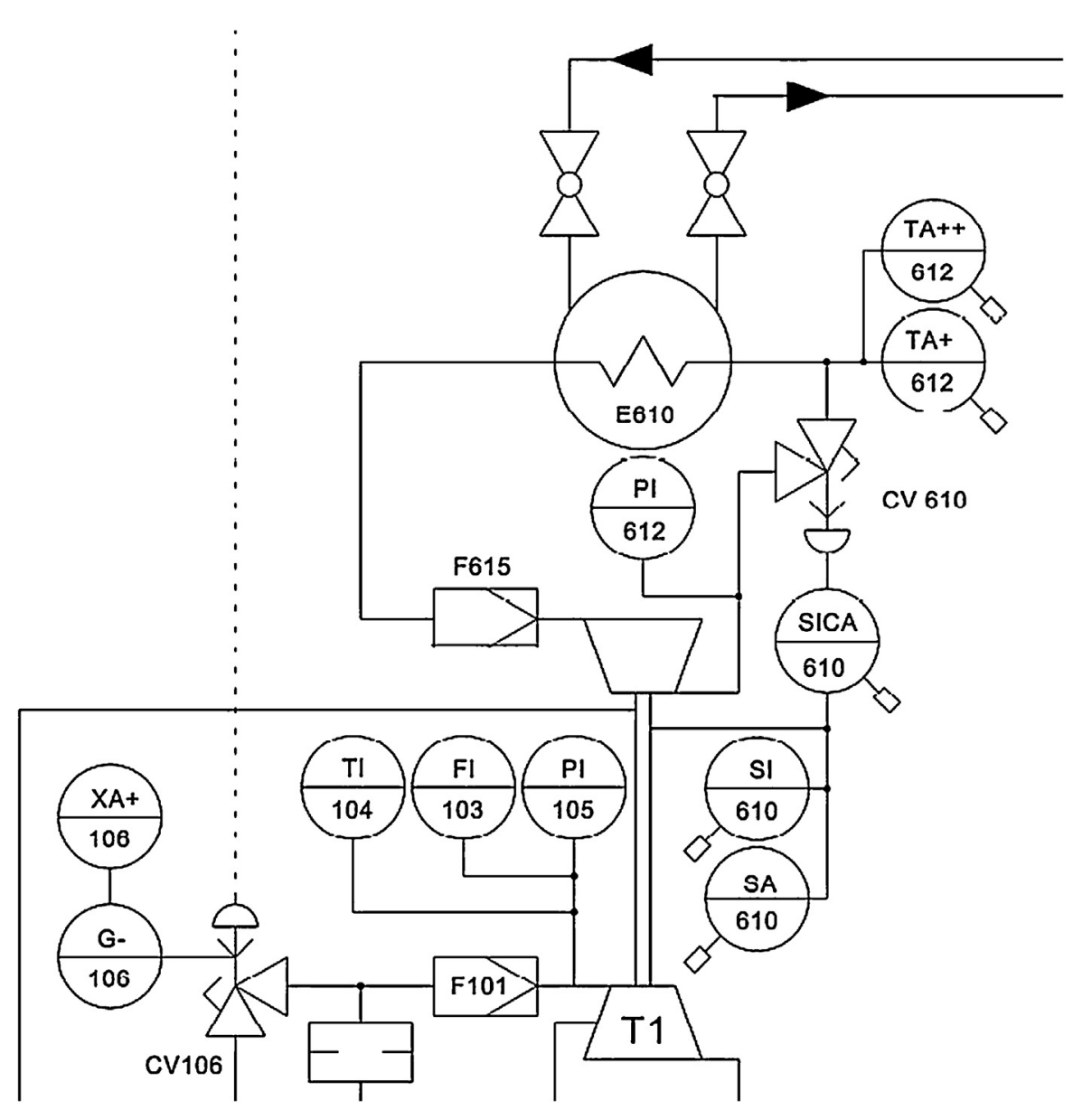

Fig. 2. Plant section in P\&ID.

As can be seen in the P\&ID in Fig. 2, the filter unit F101 is connected with the actuator CV610 by a pipe. Similarly, the filter unit F615 is connected by a pipe with the heat exchanger E610. Physical and electrical connections are modelled in CAEX with the concept of InternalLinks (IL). The red rectangles with the red dotted lines in Fig. 3 highlight the connectivity of CAEX plant items in a graphical manner. In CAEX, the connections between elements of the "InstanceHierarchy" are modelled by use of InternalLinks.

The topology model is built by defining classes and by instantiating objects from these classes. Classes which are used typically in process facilities include valve, sensor, measuring point, pump, turbine, heat exchanger and process equipment such as vessel, absorber and heat exchanger. In the case of valves and sensors, different types of these assets can be found in plants, as shown in Table 1.

Automation system users usually have their own naming conventions for instrument and alarm tags. However, it is possible to identify the related alarm tag in the automation system and alarm log with the help of the symbols and name tags of the items in the P\&ID. For example in the Plant 1 case study to be discussed below, a pressure sensor in plant section 42 is represented by PI and the number 105 in the P\&ID. The defined alarm tag for this specific alarm is 42PI105_ai. Each InternalElement connects the alarm tags with the information about the type of this item as shown in Table 2.

Summarizing, the CAEX model combines information about the topology, the sensors and actuators, the related alarm tags, and the types of the devices. The topology is represented by the InternalLinks between the Elements which show all physical and functional connections. The alarm tags are identical to the tags from the alarm system and associated to their elements. The type of the
Table 1

Valve and sensor SystemUnitClasses in CAEX model.

\begin{tabular}{ll}
\hline Class & Type \\
\hline & Controllable Valves: \\
& $\bullet$ On/Off Valve \\
& $\bullet$ Control Valve \\
& $\bullet$ Control Valve (FailOpen) \\
& $\bullet$ Control Valve (FailClosed) \\
Ualve & Uncontrollable Valves: \\
& $\bullet$ Pressure Control Valve \\
& $\bullet$ Check Valve \\
& $\bullet$ Revolution Speed \\
& $\bullet$ Temperature \\
& $\bullet$ Flow \\
& $\bullet$ Pressure \\
& $\bullet$ Quantity \\
& $\bullet$ Pressure Difference \\
Sensor & $\bullet$ Level \\
\hline
\end{tabular}

Table 2

Content of InternalElement.

P\&ID:
InternalElement:
PI105
•Role: Sensor
•Class: Sensor/Pressure
•Interfaces: 42PI105_ai




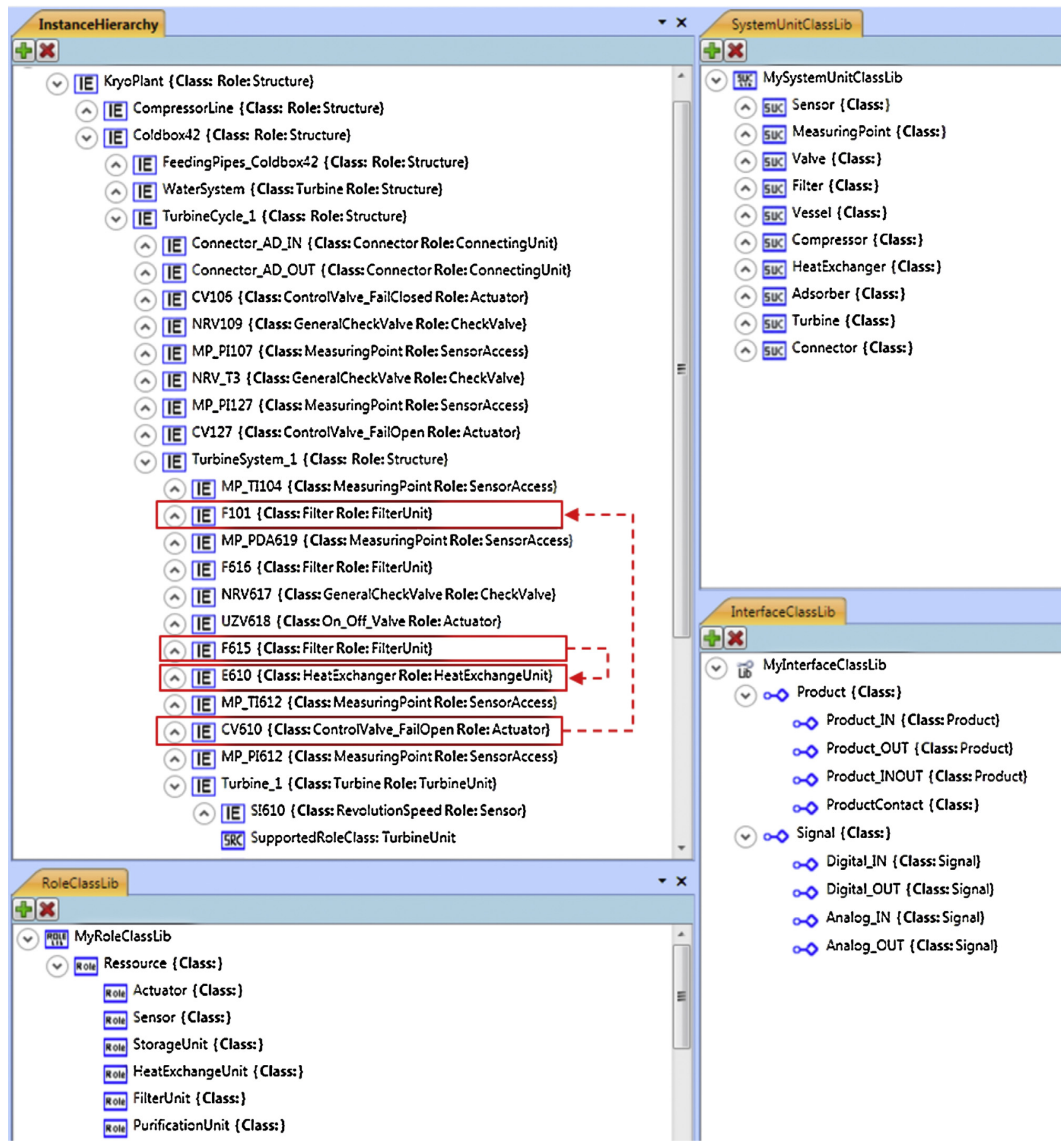

Fig. 3. Plant section as CAEX model. (For interpretation of the references to color in text, the reader is referred to the web version of the article.)

device can be found by following the references to the SystemUnitClassLib and RoleClassLib. All plant models have the same structure of libraries, which allows a general approach on processing the CAEX models.

Serving as examples, two industrial process facilities have been examined in detail with this approach, called "Plant_1" and "Plant_2" in the following. Figs. 4 and 5 show parts of the P\&IDs belonging to the plants. The CAEX model of Plant_1 model consists of around 100 sensors and 50 controllable valves, seven turbines and four compressors, the latter being equipped with filters and check valves. The model of Plant_2 comprises 25 sensors and 15 controllable valves and several compressors and motors.

\section{Alarm history and alarm logs}

The alarm logs for these studies contain the recorded alarm items caused by disturbances while the plants were in normal operation mode. Table 3 shows an original alarm log from the Plant_1 example. In the table, Kryo-Anlage refers to the cryogenic plant area, Austritt refers to an exit pressure, Lagergas is gas storage and is showing differential pressure alarms, and Strom vor Regelventil refers to a pressure alarm in a location before a flow control valve. The eventtime shows date and time with millisecond resolution when the alarm is registered. The logs reveal numerous alarms which are registered in the same millisecond. This 


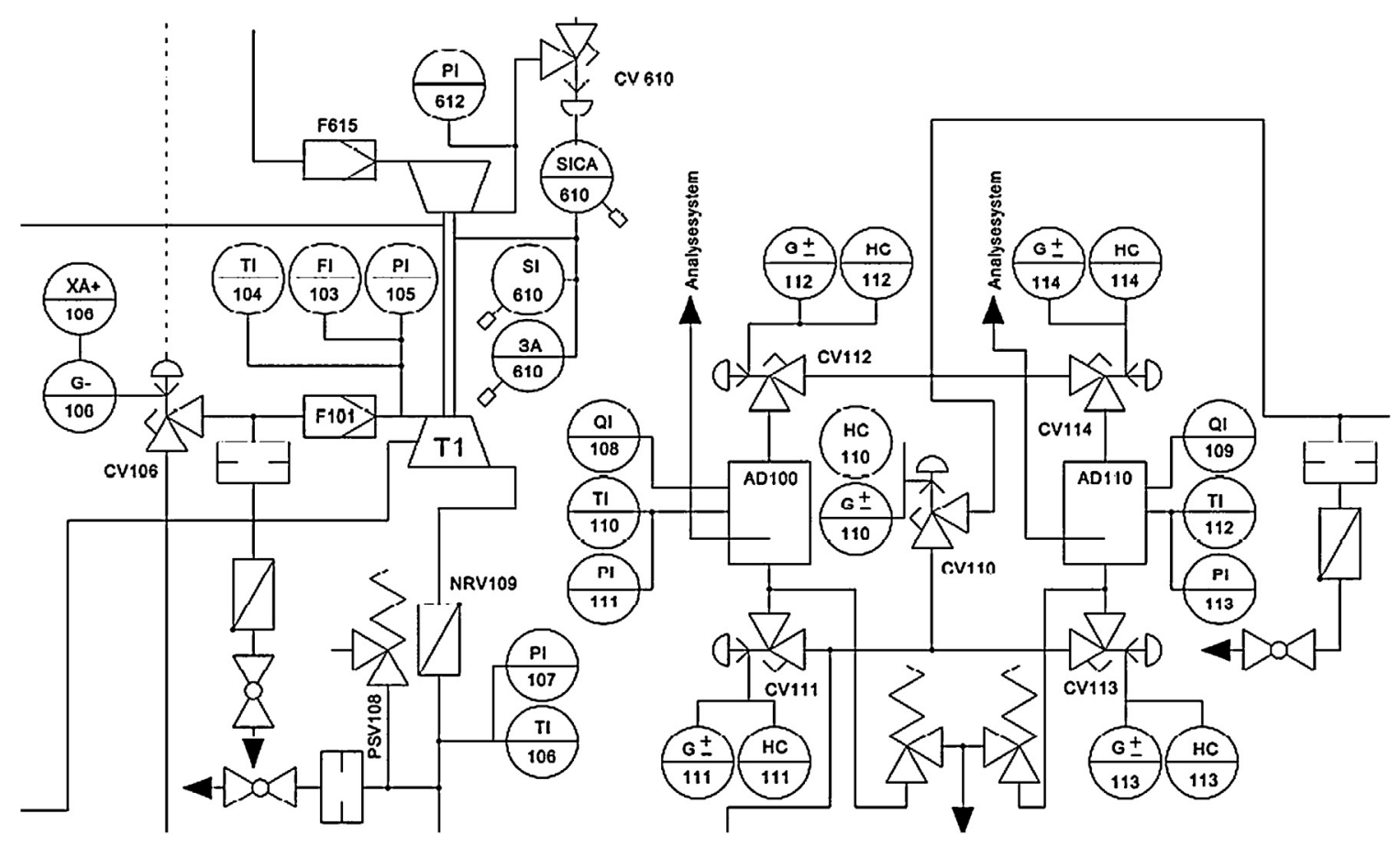

Fig. 4. Section from the P\&ID for Plant_1.

artefact can be explained by the resolution of the alarm management system and the cycle time of the control system. For this reason, it is not reliable to group alarms based only on time stamp even if these alarms may appear to have happened simultaneously. The alarmtext offers useful information for the operator because it describes the device which has caused this entry in the alarm log. Alarmtag is the label of the device which caused the alarm. Area describes the part of a plant where the alarm is generated. Severity indicates the impact and allowed reaction time as defined during the alarm design. Examples of alarm severity in Plant_1 are Major, Minor, No Alarm and Invalid. The attribute Status often shows the direction in which the alarm variable has deviated if it is possible to be determined. For example, a temperature variable might exceed or fall below the required process conditions which is then displayed by High or Low for a normal alarm and HIHI or LOLO for a second stage (more urgent) alarm. HWLIMIT may be shown if the measurement range of a sensor is exceeded. STATE indicates an alarm status which is based on a logic function in the automation system. For example a general alarm can be triggered if the system detects a pump shutdown.

Unfortunately, there is no common alarm terminology used in industry and the alarm logs are plant-specific, which requires a semantic mapping before the alarms can be evaluated automatically. For example, the alarm logs of Plant_2 show 13 levels of alarm severity and Plant_2 example also has additional Status values such as MALFUNCTION or ERROR.

\section{Interrelation rules}

The aim of this paper is to reduce the number of different single but related alarms by a method of grouping alarms. Alarms which are obviously related to each other are grouped to reduce the total number of alarms shown to the operator. This section describes the syntax and required attributes for designing the required rules for grouping.

\subsection{Syntax of interrelation rules}

The approach of combining alarm history with plant topology to reduce the quantity of alarms is supported by a rule base. The interrelation rule base sets the conditions to group alarms with a common cause. This involves information from the previously described alarm logs and plant topology model. Fig. 6 illustrates the general method for defining rules.

Rules apply to (i) the elapsed time between alarms, (ii) the types of alarms that can be grouped, (iii) the type of connection (e.g. a process product line or a signal connection), and (iv) the number and types of items of equipment in the process that can be in between the locations of the alarms. Rules about the types of alarms that can be grouped are based on process knowledge and first principles understanding. Other aspects such as the allowed types and number of items between the alarms locations are tuneable parameters which affect the extent of the alarm grouping. Table 4 shows the syntax of interrelation rules.

Table 3

Alarm log of Plant_1.

\begin{tabular}{|c|c|c|c|c|c|}
\hline EVENTTIME & ALARMTEXT & ALAMTAG & AREA & SEVERITY & STATUS \\
\hline 2010-11-09 16:30:18.878 & Austritt HD E4 & 42PI439_ai & Kryo-Anlage & MINOR & $\mathrm{HIGH}$ \\
\hline 2010-11-09 16:30:20.178 & JT-Strom vor Regelventil & 42PI401_ai & Kryo-Anlage & MINOR & $\mathrm{HIGH}$ \\
\hline 2010-11-09 16:30:29.677 & JT-Strom vor Regelventil & 42PI401_ai & Kryo-Anlage & MAJOR & HIHI \\
\hline 2010-11-09 16:30:31.877 & Austritt HD E4 & 42PI439_ai & Kryo-Anlage & MAJOR & HIHI \\
\hline 2010-11-09 16:30:31.910 & Lagergas Anspeisg T7 & 42PDA679_bi & Kryo-Anlage & MAJOR & STATE \\
\hline 2010-11-09 16:30:31.910 & Lagergas Anspeisg T6 & 42PDA669_bi & Kryo-Anlage & MAJOR & STATE \\
\hline 2010-11-09 16:30:44.877 & Austritt HD E4 & 42PI439_ai & Kryo-Anlage & MAJOR & HWLIMIT \\
\hline
\end{tabular}




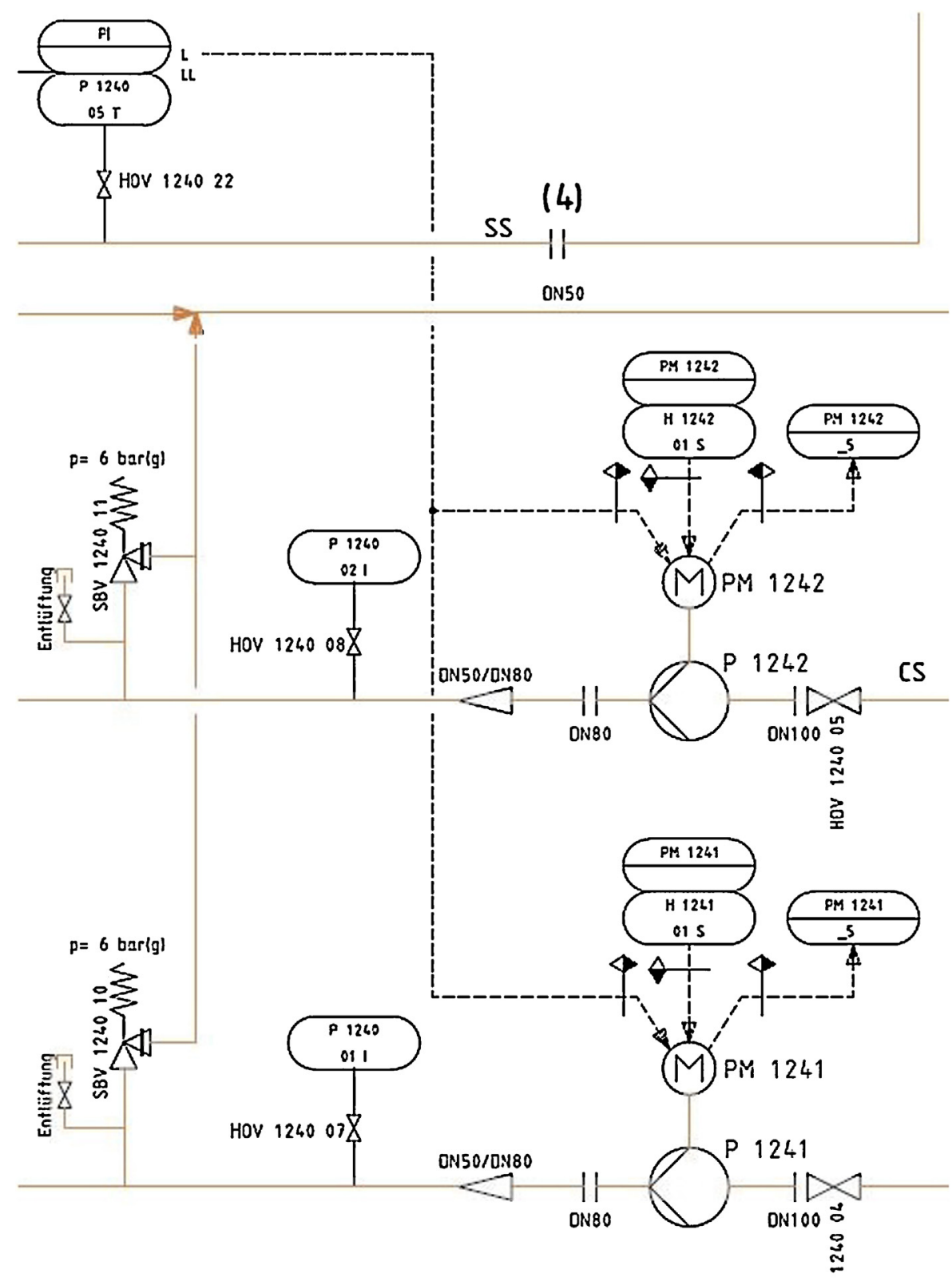

Fig. 5. Section from the P\&ID for Plant_2.

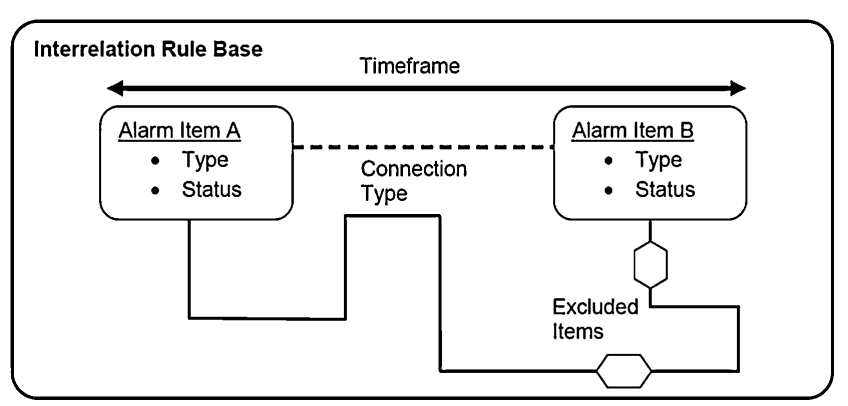

Fig. 6. Approach for interrelation rules.
The analysis examines every possible pair of two alarm messages to determine whether there is an interrelation between them. For illustration, two pressure alarms probably have the same cause if they also comply with some further conditions such as occurring in a short time interval and having a physical process path connecting them. Some excluded items are not allowed on this path. For example a pump should not be allowed on the process path between two pressure alarms because pressure alarms either side of a pump could be independent from each other. If two alarms trigger the rule, then they can be grouped into one collective alarm problem.

Table 4

Syntax of the interrelation rule.

\begin{tabular}{|c|c|c|c|c|c|c|}
\hline Type: Alarm A & Type: Alarm B & Time frame & Status match & Connection type & Max. no. of items & Allowed Item on PATH \\
\hline Type A & Type B & $X s$ & YES/NO & Product/Signal & $0-X$ & Device $x$ \\
\hline
\end{tabular}


Table 5

Settling time for loop types.

\begin{tabular}{ll}
\hline Loop type & Settling time \\
\hline Pressure & $100 \mathrm{~s}$ \\
Liquid flow & $30 \mathrm{~s}$ \\
Temperature & $360-600 \mathrm{~s}$ \\
Steam or gas flow & $300 \mathrm{~s}$ \\
Level (tight level control) & $100-200 \mathrm{~s}$ \\
\hline
\end{tabular}

\subsection{Rule properties}

\subsubsection{Alarm type}

Any rule requires the type of two alarm items, such as pressure alarm or temperature alarm. The use of alarm types allows a universal application of the approach and is needed because the tag naming convention varies from plant to plant. Rules are based on the type of an alarm instead of involving the real name of an alarm device. Rules that refer to the role, system unit and interface libraries of the plant model can be easily transferred to other process facilities if they use identical libraries. Information about alarm tag and alarm type is then linked together in the CAEX plant model.

\subsubsection{Time frame}

Two alarms must not exceed a specified elapsed time to be candidates for grouping. The time stamp is taken from the alarm log as an output of the process control system. The allowed time frame between any two alarms with a common cause depends on the alarm type because it allows time for fault propagation through parts of the plant as well as time for control system loop cycles. Different time frames are defined for each combination of alarm type. However it is also reasonable to adjust these to existing plant properties and alarm systems, because the elapsed time before a deviation in a process variable causes an alarm message depends on how the alarm thresholds have been set in the automation system. In general, a longer time frame would include more alarm messages in the alarm group, but alarms that occur in a shorter time frame are more likely to have the same root cause. Therefore the timeframe is an important design parameter in the tuning of the interrelation rules.

ANSI/ISA 18.2 [3] gives recommendations for a delay time. Delay time is an elapsed period of time before the automation system indicates an alarm after the variable has passed the alarm threshold. Its purpose is to avoid chattering alarms caused by intermittent excursions of the alarm threshold. However, the timeframe parameter in the interrelation rules has a different purpose. It takes into account that processes have dynamics such that alarms which are related may not have the same time stamp. Typical closed loop settling times for various process parameters are shown in Table 5 [19] and these may be used as default time frames in the interrelation rules.

The settling times used in this approach provide an estimate about how quickly disturbances (which might raise an alarm) will probably propagate to neighbouring tags (and raise alarms there). These settling times can serve as initial values for the time frames in the rules, however, they need to be confirmed by practical experience.

\subsubsection{Status}

The status of an alarm item gives information about the event that caused the alarm in the automation system. The status from the alarm logs can be indicating a high, low alarm or a hardware failure, for example. The interrelation rules compare the status of two alarm messages to further establish a link between them. If two pressure sensors are triggering alarms with a low status message, because the pressure dropped below the low pressure alarm threshold, then the likelihood is strong that there is an interrelation. In contrast to this, the alarm status of a level alarm and a valve failure alarm cannot match, because the status descriptions (low/high for the level and error for the valve) do not overlap. A level alarm and a valve alarm may be related or may not, so the status match rule would not be used in that case and would leave the decision about a match to other rules. If two alarms of the same type (e.g. "temperature") belong to the same group, they will most probably have identical alarm status (e.g. high alarm "high boiler temperature" and high alarm "high boiler outlet temperature"). However, a temperature low alarm at one part of the plant and a temperature high alarm somewhere else are usually considered as separate alarms. On the contrary, alarms of different type with different alarm status can likely belong to one group, e.g. a low level alarm ("low cooling water level") and a high temperature alarm ("high boiler temperature"), which stem from the same physical cause. The status match requirement can be changed by the user in the rule base if desired.

\subsubsection{Connection type}

After finding two candidates for the same alarm group the plant topology is searched for a connecting path between them. Connections can be physical connections via pipes or connections through signals from the process control system. Other possible connections like hydraulic, pneumatic or electrical connections are typically not described in P\&IDs and would require a future extension both of the available CAEX modelling tools and of the interrelation rules.

Each interrelation rule describes what type of connection may be considered in exploring the connecting path. The connecting path lists all modelled items which can be found on the route between the two alarm candidates. While there may be routes through the process topology between two alarm candidates, only those without an excluded item are classed as connecting paths. For example a pump may not be allowed between two pressure alarms because a pump divides the process flow line into two pressure zones in which alarms may not be related.

A further interrelation rule sets a maximum number of items of any kind on the connecting path. A consequence of the rule is that it is possible to have a sensor on the connecting path which is not triggering an alarm. Such non-alarming sensors have to be handled carefully. One reason can be that the non-alarming sensor has broader alarm thresholds than the devices with alarms. Its alarm would then only be activated if the process deviation increases still further. In this case the sensor should not be defined as an excluded item. On the other hand a third sensor between two triggered sensor alarms could indicate two different alarm groups. This case leads to a rule which would exclude a third sensor on the connecting path. Both rules are reasonable depending on the properties of the alarm system. Such a case indicates the need for a rule editor which engineers can use to tune and extend the rule-based system and to optimize the alarm groups.

\section{Implementation}

The combination of plant topology models (Section 2), individual alarm messages (Section 3) and interrelation rules (Section 4) has been implemented in a software prototype which analyses a given alarm log file and presents the results in an appropriate tree structure. This tree structure presents a compact alarm group at the top level and allows exploration through the alarm groups to subsequently show the single alarm messages in each branch.

\subsection{Rule editor}

A rule editor is required for the first application of the approach. Therefore the prototype offers an interface (Fig. 7) where 


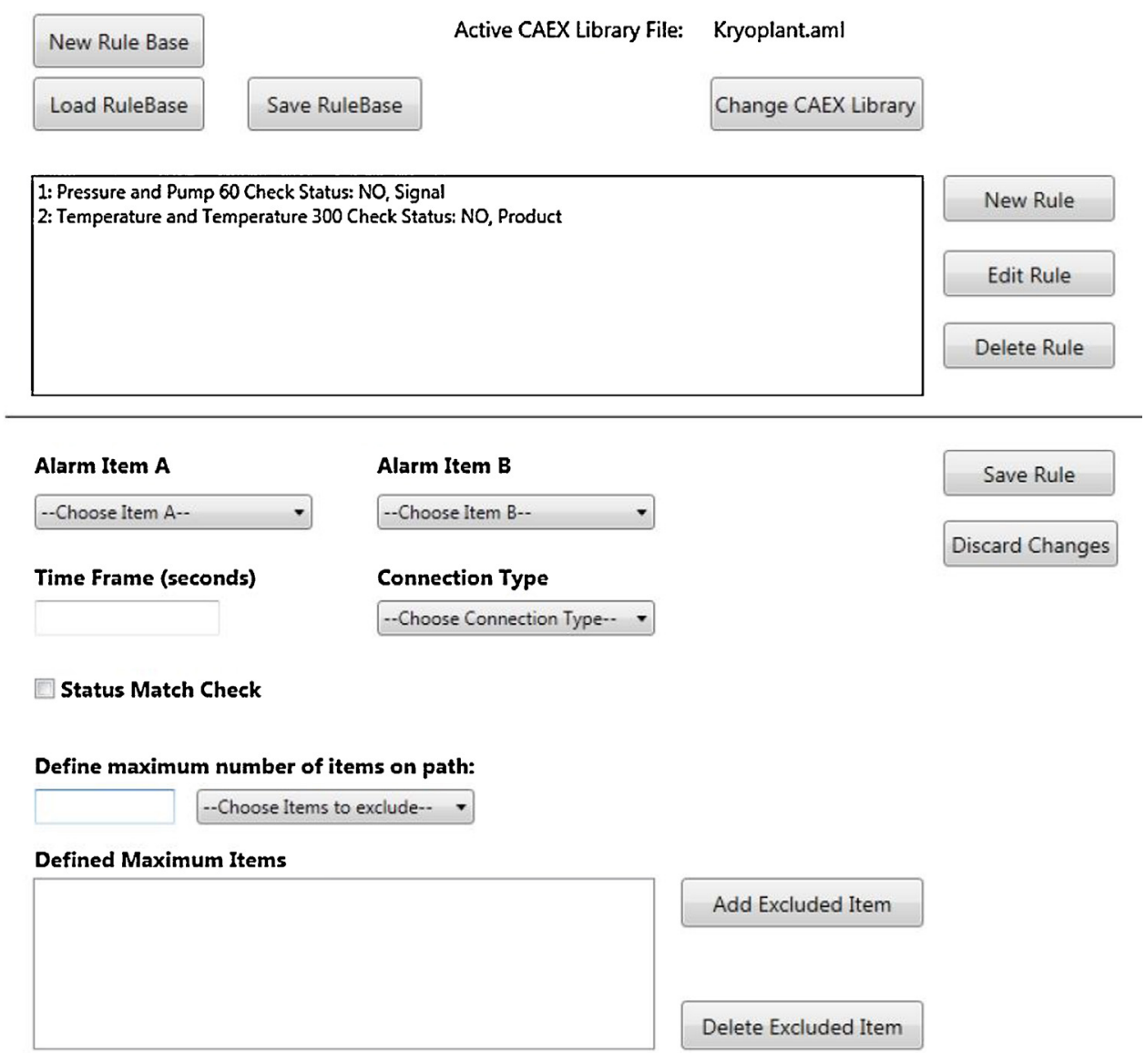

Fig. 7. Rule editor.

interrelation rules can be designed and adjusted with the attributes described in Section 3. The structure and contents of permitted rules are defined in an XML schema developed as part of the project which checks them for correct formulation. Thus, the rules can easily be modified and extended by the user through the rule editor. Rule 2 in the example in Fig. 7 shows that both alarms must be of type Temperature and that status checks are to be used. The time frame is $300 \mathrm{~s}$. The connection type is to be though product lines and no excluded items are being specified. The pull down menus are populated from the CAEX plant model so that the user can apply the generic rules to the specific plant. A group of rules can be saved as a rule base.

\subsection{Analysis algorithms}

From the rule base, the algorithm searches for two alarm log items which are candidates for grouping, according to the rule base. Fig. 8 illustrates this process in a flow chart. After two candidates for alarm grouping meet the conditions of the type, time frame and status rules, the plant topology is searched for a physical or functional connection (dotted lines in Fig. 3, corresponding to the physical connections via pipes which are visible in Fig. 2) to check the interrelation between them (Fig. 9).

As a result of the analysis, the alarm log is presented to the user in a tree structure. Fig. 10 depicts the user interface of the software prototype. At the top, basic information like plant, rule base as well as the total number of alarms and number of alarm groups after processing are displayed. The lower part contains the same alarms as the original alarm list but now structured according to the groupings detected by the algorithm. The alarms which occurred first and last are in bold font. Other related alarms in the same group can be seen by clicking on the first alarm of the group; these are shown in a lighter grey font. It should be noted that the alarms in bold are not necessarily the root cause for the disturbance, they only mark the cornerstones of the time interval of the alarms in this group.

\section{Application of the approach}

The reduction of the number of alarms has been evaluated on the Plant_1 and Plant_ 2 case studies. The section reports the results, and also comments on the effects of tuning of the parameters on the interrelation rules. Several rule bases have been created and examined regarding their effectiveness in alarm reduction.

The evaluation has been based on five logged alarm series from both case studies, three alarm logs from Plant_1 and two alarm logs from Plant_2. The sample times of these alarm series range from $10 \mathrm{~s}$ up to $2 \mathrm{~h}$ as shown in Table 6 . The number of single alarm messages in this series ranges from 30 up to 478 .

These alarm history files have been processed with six different rule bases. Each rule base contains general rules that can be applied on the two case studies.

Table 7 lists the absolute and relative reduction from single alarm messages to comprehensive alarm groups for each

Table 6

Alarm log files.

\begin{tabular}{llc}
\hline Facility alarm log & Total duration & Number of alarms \\
\hline Plant_1_A & $2 \mathrm{~h}$ & 478 \\
Plant_1_B & $1 \mathrm{~h}$ & 50 \\
Plant_1_C & $1 \mathrm{~h} 5 \mathrm{~min}$ & 28 \\
Plant_2_A & $10 \mathrm{~s}$ & 130 \\
Plant_2_B & $45 \mathrm{~min}$ & 30 \\
\hline
\end{tabular}




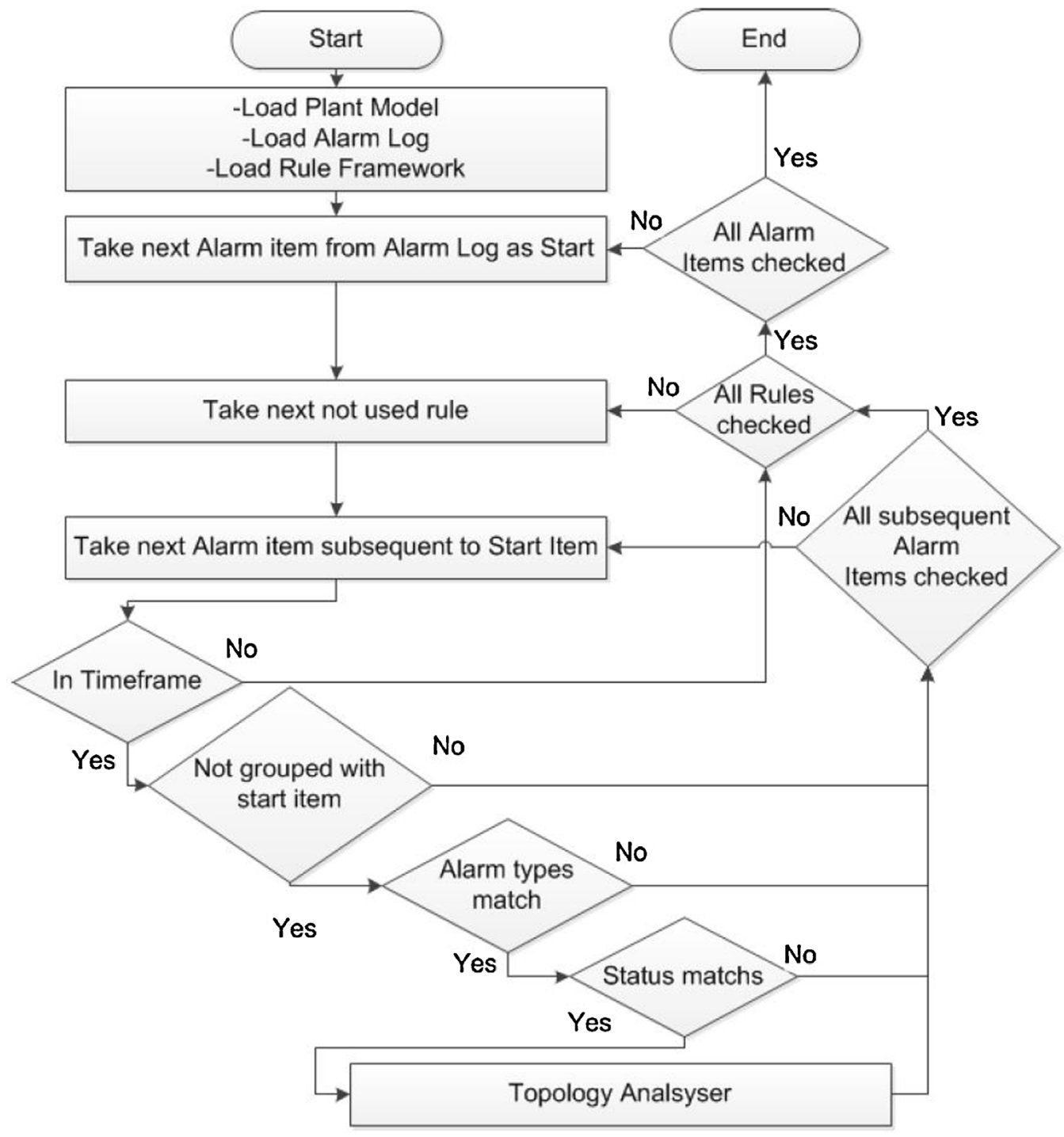

Fig. 8. Flow chart - finding candidates for alarm grouping.

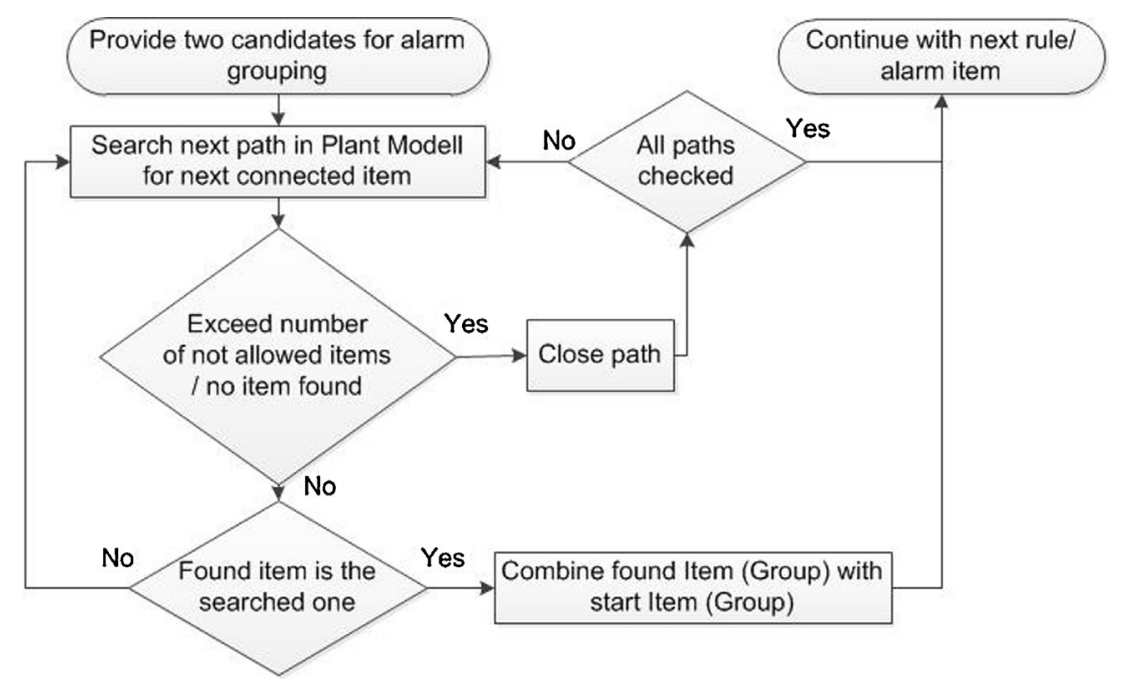

Fig. 9. Flow chart - finding connection in plant topology. 


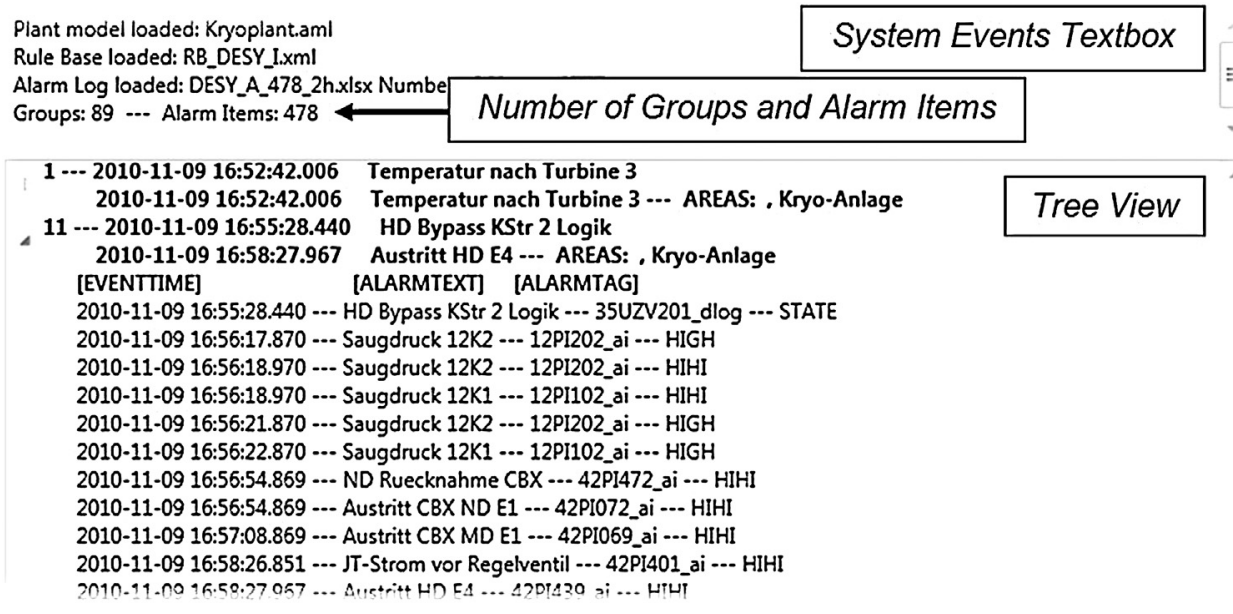

Fig. 10. Presentation of results of alarm grouping process.

Table 7

Results of alarm grouping.

\begin{tabular}{|c|c|c|c|c|c|c|c|}
\hline \multirow[t]{2}{*}{ Facility alarm log } & \multirow[t]{2}{*}{ Number of alarms (in model) } & \multicolumn{6}{|c|}{ Reduction of alarms to groups: total number (percentage) } \\
\hline & & A & B & $\mathrm{C}$ & $\mathrm{D}$ & $\mathrm{E}$ & $\mathrm{F}$ \\
\hline Plant_1_1 & 478 & $80(83 \%)$ & $60(87 \%)$ & $70(85 \%)$ & $66(86 \%)$ & $49(90 \%)$ & $49(90 \%)$ \\
\hline Plant_1_2 & 50 & $20(60 \%)$ & $16(68 \%)$ & $19(62 \%)$ & $17(66 \%)$ & $14(72 \%)$ & $13(74 \%)$ \\
\hline Plant_1_3 & 28 & $17(39 \%)$ & $9(68 \%)$ & $15(46 \%)$ & $16(43 \%)$ & $7(75 \%)$ & $7(75 \%)$ \\
\hline Plant_2_1 & 47 & $9(81 \%)$ & $9(81 \%)$ & $9(81 \%)$ & $9(81 \%)$ & $9(81 \%)$ & $5(89 \%)$ \\
\hline Plant_2_2 & 24 & $6(75 \%)$ & $6(75 \%)$ & $6(75 \%)$ & $6(75 \%)$ & $6(75 \%)$ & $3(88 \%)$ \\
\hline Reduction in \% & & $67.6 \%$ & $75.8 \%$ & $69.8 \%$ & $70.2 \%$ & $78.6 \%$ & $83.2 \%$ \\
\hline
\end{tabular}

combination of alarm log and rule base shown in Table 8. The last row of Table 7 shows the average reduction in percent for each rule base relating to all alarm logs.

Rule base A defines the strictest interrelation rules allowing no excluded items, setting a moderate time frame and having the option for status match checking enabled. The maximum number of controllable valves between a sensor and a valve, pump or turbine is increased from zero to two in rule base $B$. This resulted in an increased reduction of $75.8 \%$ with rule base B compared to $67.7 \%$ with rule base $A$. The compromise for every variation to the rules is to find rules that group a number of alarms together but still preserve the difference between separate disturbances. Therefore a higher number of excluded items are chosen in rule base $\mathrm{B}$, which also has to be adjusted to the current plant and alarm system. Rule base B allows one sensor of the same type between two alarm sensors. This increase is reasonable as different alarm

Table 8

Overview rule bases A-F.

\begin{tabular}{ll}
\hline Rule base A & Low value for time frame attribute \\
& 0 controllable valves between sensor and actuator \\
allowed & 0 sensor between two sensors allowed \\
& Checking status match enabled for sensors with \\
same alarm type & Rule base A with: \\
& 2 controllable valves between sensor and actuator \\
allowed & 1 sensor between two sensors allowed \\
Rule base B & 1 Sensor between two sensors allowed for a short \\
& time frame \\
& 0 Sensor between two sensors allowed for a longer \\
Rule base C & time frame \\
& Rule base A with: time frame is doubled \\
& Combination of rule bases B and D \\
Rule base E without checking status match
\end{tabular}

thresholds are likely to leave single sensors deactivated while other alarms are already activated. This setting depends on the alarm system because different alarm systems do not have the same alarm thresholds.

Rule base $C$ combines the restrictive rule base $A$ and the less restrictive rule base $B$ by having a short time frame in which no sensor is excluded from the connecting path and a longer time frame with a limit of one allowed sensor. The reduction in this case $69.8 \%$ compared to $67.6 \%$ of rule base $A$ indicates that the increase of allowed controllable valves has a greater influence than increase the number of allowed sensors and hence the setting of rule base $B$ is further evaluated in upcoming rule base $E$.

The influence of the defined time frame on the interrelations is researched in rule base $D$. All values for time frame of rule base $A$ are doubled in this setting. The result is an average reduction of $70.2 \%$ compared to $67.6 \%$ of rule base A. The optimal setting for the time frame depends on the alarm type and also on the plant process. The adjustment of these settings has to be done with the experience of the plant operators and process engineers but the time frame in rule base $\mathrm{D}$ is reasonable as after the analysis still separate alarm groups remain which describe different disturbances.

The combination of a doubled time frame in rule base D and increased number of excluded items in rule base $B$ resulted in rule base $\mathrm{E}$. The reduction is with $78.6 \%$ in this case significant higher than the $67.6 \%$ reduction of rule base $A$ as it combines the advantages of both previous rule bases. Further increase of the time frame did not result in further grouping of alarms. This provides evidence that the time frames chosen here are appropriate for the particular process and size of the plant under consideration.

All previous rule bases had the check for matching status between two similar sensors enabled. Rule base $\mathrm{F}$ is identical to rule base $\mathrm{E}$ but the option to check for a matching status is disabled to research the impact of this option. The results in Table 7 show a maximum average reduction of $83.2 \%$ which is the highest 
reduction of all, and a $4.6 \%$ increase compared to rule base E. This shows that the status match restriction should be relaxed. Further analysis revealed that, with the current implementation, the status check condition is only fulfilled when the two states are exactly identical, based on a character string comparison. Therefore alarms with the status "HIGH" and "HIHI" (very high) are considered to be of different type with all rule bases except of rule base $\mathrm{E}$ and are therefore not grouped.

The Plant_2 alarm logs for example contain alarms with a MAX1 or MAX2 status. Those alarms are not grouped together, although they comply with all other conditions of the interrelation rules. In contrast to this rule base $\mathrm{F}$ allows grouping alarms with high and low alarm status together because the status check option is disabled. The deactivation is not reasonable in every scenario, however. The possible average reduction should be found between the results of rule bases $\mathrm{E}$ and $\mathrm{F}$ with $78.6 \%$ and $83.2 \%$ if the analysis is able to determine which status attributes are indicating an interrelation, e.g. high and HIHI should be identified as same direction of deviation.

The trends discussed above show that the rules are operating as expected in the given case studies. They show that strict tuning of the rules such as in rule base A leads to lower levels of alarm grouping, as would be expected. Allowing longer time frames or more equipment items on the connection paths leads more alarms to be grouped and hence a larger reduction in the number of alarms.

It was not possible for the academic investigators to create alarms in the industrial facility. Moreover, the true causes of the alarms in the alarm logs (and hence the number of separate alarm groups) were not known. There is thus no quantitative benchmark available by which to evaluate the results. Nevertheless, the opinion of the authors is that rule base $\mathrm{E}$ is the most reliable set of rules with a combination of an extended value for the time frame and moderate restrictions by excluded items. This rule base uses a status match to separate sensor alarms which do not have the same kind of deviation. The rule base can be adjusted to other plant models or alarm systems but the results of the case studies suggest that rule base $\mathrm{E}$ is the best default option regarding to the level of reduction and reasonable alarm grouping.

The restrictions made by the authors regarding the rule properties as well as the combination of rules can and have to be adjusted by the operator during operation. They are recommendations to show that the proposed method can be applied successfully to different processes to reduce the total amount of alarm visualized to the operator.

\section{Conclusion and outlook}

The paper has demonstrated an automated method for grouping of alarms in the alarm log of a process automation system. Key advances on the current industrial state of the art are (i) that it uses process connectivity information which is not present in hierarchical functional structure of the automation system, and (ii) it uses tuneable interrelation rules based on basic process knowledge and insights.

The use of rules which contain knowledge of plant connectivity is a promising approach for the reduction of the number of alarms shown to the operator. Additionally, a rule editor allows tuning of the alarm grouping by operators or process control engineers, as would be needed for adoption in an industrial automation system. The construction of the interrelation rules based on libraries according to the new XML schema offers a general approach. Interrelation rules specify the search criteria for alarms likely to have the same cause. The rules consider temporal proximity, type of the alarm and spatial and functional structure which can be found in the plant topology. These rules allow the grouping of individual alarm messages into fewer groups that give a more comprehensive insight into alarm problems. The concept has been implemented in a software prototype which manages the alarm log, plant model and interrelation rules and presents the grouped alarms in an interactive alarm display. The alarms are not deleted from the alarm logs. Rather, it is the same alarm log but structured hierarchically. The result is a compact alarm display with fewer alarm messages visible on the top level but a higher information density. The application of the approach on two case studies resulted in a successful reduction of alarms. The grouping of related alarms has reduced the total amount of single alarm messages between 70 and $80 \%$ depending on the tuning of the interrelation rules. This is a promising approach to support the operators in finding the root cause for process alarms.

The approach described here should not be understood as a stand-alone activity which is sufficient to completely analyze and evaluate the alarm system of a plant. Instead, the proposed method can, and should be, applied within an alarm management system improvement project, with proper management of change. The proposed method can only provide an indication regarding alarms which might have a common cause. The groups of alarms should be analyzed (e.g. within a PHA (Process Hazard Analysis) assessment) by a human expert whether there is a proper rationale to group these alarms. The authors plan to integrate their approach into such a broader context.

Another direction of our future work will be to analyze how the concept can be implemented in a process control system, with access to on-line alarm information instead of alarm logs, for realtime grouping of alarms.

\section{Acknowledgements}

Markus Schleburg gratefully acknowledges funding from the Erasmus Student Mobility grant for study at Imperial College London. Nina Thornhill gratefully acknowledges funding from $A B B$ and the Royal Academy of Engineering for the ABB/RAEng Research Chair in Process Automation at Imperial College London.

\section{References}

[1] M. Blanke, M. Kinnaert, J. Lunze, M. Staroswiecki, Diagnosis and Fault-Tolerant Control, 1st ed., Springer, Berlin, 2003, ISBN 3-540-01056-4.

[2] I. Izadi, S.L. Shah, T. Chen, Effective resource utilization for alarm management, in: 49th IEEE Conference in Decision and Control, 2010, pp. 6803-6808.

[3] American National Standards Institute (ANSI), ANSI/ISA-18.2, Management of Alarm Systems for the Process Industries, New York, 2009.

[4] Engineering Equipment and Materials Users' Association (EEMUA), EEMUA 191, Alarm Systems - A Guide to Design, Management and Procurement, London, 2007.

[5] N.A. Adnan, I. Izadi, T.W. Chen, On expected detection delays for alarm systems with deadbands and delay-timers, Journal of Process Control 21 (2011) 1318-1331.

[6] J. Ahnlund, T. Berquist, An alarm reduction application at a district heating plant, in: IEEE Conference on Emerging Technologies and Factory Automation, 2003, pp. 187-190

[7] R. Candy, J. Taisne, Advanced alarm processing facilities installed on Eskom's energy management system, in: IEEE PES Power Africa 2007 Conference, Johannesburg, South Africa, July 16-20, 2007.

[8] O. Aizpurúa, R. Caballero, R. Galán, A. Jiménez, A new methodology for massive alarm management system in electrical power administration, in: 7 th Latin American and Caribbean Conference for Engineering and Technology, Venezuela, June 2-5, 2009.

[9] H.A. Gabbar, R. Datu, H. Hayashi, D. Akinlade, A. Suzue, M. Kamel, Development of root cause \& consequence analyzer for intelligent fault diagnosis, in: SICEICASE International Joint Conference, Busan, Korea, October 18-21, 2006.

[10] J. Thambirajah, L. Benabbas, M. Bauer, N.F. Thornhill, Cause-and-effect analysis in chemical processes utilizing XML, plant connectivity and quantitative process history, Computers and Chemical Engineering 33 (2009) 503-512.

[11] International Electrotechnical Commission, IEC 62424, Representation of Process Control Engineering - Requests in P\&I-Diagrams and Data Exchange Between P\&ID Tools and PCE-CAE Tools, 2008.

[12] B.R. Hollifield, E. Habibi, The Alarm Management Handbook - A Comprehensive Guide, 2nd ed., ISA, Houston, 2010, ISBN 978-1936007554. 
[13] I. Izadi, S.L. Shah, D.S. Shook, S.R. Kondaveeti, T. Chen, A framework for optimal design of alarm systems, in: Proceedings of the 7th IFAC Safeprocess, Barcelona, 2009.

[14] F. Yang, S.L. Shah, D. Xiao, T. Chen, Correlation analysis of alarm data and alarm limit design for industrial processes, ISA Transactions 51 (2012) 499506.

[15] M. Fedai, U. Epple, R. Drath, A. Fay, Metamodel for generic data exchange between various CAE systems, in: Proceedings 4th Mathmod, Vienna, 2003, pp. 1247-1256.
[16] M. Fedai, R. Drath, CAEX - a neutral data exchange format for engineering data, ATP International Automation Technology 3 (1) (2005) 43-51.

[17] M. Barth, M. Strube, A. Fay, P. Weber, J. Greifeneder, Object-oriented engineering data exchange as a base for automatic generation of simulation models, in: Proceedings 35th IECON Porto, 2009, pp. 2465-2470.

[18] Anon, AutomationML Files. https://www.automationml.org/o.red.c/dateien. html (accessed 20.01.13).

[19] N.F. Thornhill, M. Oettinger, P. Fedenczuk, Refinery-wide control loop performance assessment, Journal of Process Control 9 (1999) 109-124. 